\title{
Imaging of neuroinflammation due to repetitive head injury in currently active kickboxers
}

\author{
Gilles N. Stormezand ${ }^{1}$ (D) Janine Doorduin ${ }^{1}$. Sandra E. Rakers ${ }^{2}$. Jacoba M. Spikman ${ }^{2}$. Joukje van der Naalt ${ }^{3}$. \\ David Vállez García ${ }^{1}$. Anouk van der Hoorn ${ }^{4}$. Chris W. J. van der Weijden ${ }^{1} \cdot$ Berry P. H. Kremer ${ }^{3} \cdot$ Remco J. Renken $^{5}$. \\ Rudi A. J. O. Dierckx ${ }^{1}$
}

Received: 28 October 2021 / Accepted: 28 January 2022 / Published online: 15 February 2022

(c) The Author(s) 2022, corrected publication 2022

\begin{abstract}
Purpose Chronic traumatic encephalopathy refers to a neurodegenerative disease resulting from repetitive head injury of participants in contact sports. Similar to other neurodegenerative diseases, neuroinflammation is thought to play a role in the onset and progression of the disease. Limited knowledge is available regarding the neuroinflammatory consequences of repetitive head injury in currently active contact sports athletes. PET imaging of the $18-\mathrm{kDa}$ translocator protein (TSPO) allows quantification of microglial activation in vivo, a marker of neuroinflammation.

Methods Eleven rank A kickboxers and 11 age-matched controls underwent TSPO PET using $\left[{ }^{11} \mathrm{C}\right]-\mathrm{PK} 11195$, anatomical MRI, diffusion tensor imaging, and neuropsychological testing. Relevant imaging parameters were derived and correlated with the outcomes of the neuropsychological testing.

Results On a group level, no statistically significant differences were detected in non-displaceable binding potential $\left(B P_{\mathrm{ND}}\right)$ using PET. Individually, 3 kickboxers showed increased $B P_{\mathrm{ND}} \mathrm{s}$ in widespread regions of the brain without a correlation with other modalities. Increased FA was observed in the superior corona radiata bilaterally. DTI parameters in other regions did not differ between groups.

Conclusion Despite negative results on a group level, individual results suggest that neuroinflammation may be present as a consequence of repetitive head injury in active kickboxers. Future studies using a longitudinal design may determine whether the observed TSPO upregulation is related to the future development of neuropsychiatric symptoms.
\end{abstract}

Keywords Repetitive head injury $\cdot$ Neuroinflammation $\cdot$ Kickboxers $\cdot$ TSPO PET

\section{Introduction}

Chronic traumatic encephalopathy (CTE) refers to a neurodegenerative disease resulting from repetitive head injury in participants of contact sports [1]. It can manifest with neuropsychiatric symptoms which may be affective

This article is part of the Topical Collection on Neurology

Gilles N. Stormezand

g.n.stormezand01@umcg.nl

1 Department of Nuclear Medicine and Molecular Imaging, Medical Imaging Center, University of Groningen, University Medical Center Groningen, Hanzeplein 1, 9700 RB Groningen, The Netherlands

2 Department of Clinical Neuropsychology, University of Groningen, University Medical Center Groningen, Groningen, The Netherlands (depression), behavioral (aggression), and/or cognitive (concentration, memory, or executive impairments). If these latter symptoms are severe, this can be qualified as dementia. The clinical presentation is correlated with different pathological stages (I-IV) [2]. Contact sporters with the APOE epsilon-4 allele have been shown to have an increased risk of developing CTE, and the severity of

3 Department of Neurology, University of Groningen, University Medical Center Groningen, Groningen, The Netherlands

4 Department of Radiology, Medical Imaging Center, University of Groningen, University Medical Center Groningen, Groningen, The Netherlands

5 Cognitive Neuroscience Center, University Medical Center Groningen, University of Groningen, Groningen, The Netherlands 
symptoms is positively linked to the cumulative dose of head impacts [3]. Although neuropsychiatric consequences of repetitive head injury have been described as early as in 1928 by Martland in boxers, it later became apparent that the disease could also affect rugby players, American football players [4], wrestlers [5], or not be related to sports [6]. Typically, the onset of the clinical syndrome is after the athletes have ended their careers [7].

Currently, a definite diagnosis of CTE can only be established post mortem. In 2015, consensus criteria became available which defined accumulation of abnormal tau proteins in neurons and astroglia around small blood vessels at the depth of cortical sulci in an irregular pattern as pathognomonic for the disease [8]. In a series of 202 former American football players, Mez and colleagues found neuropathological evidence of CTE in 177 individuals, with a prevalence of up to $99 \%$ in former National Football League (NFL) players [9], the highest professional level. Recently, diagnostic criteria have become available for the clinical diagnosis during life [10]. Although not yet part of the diagnostic criteria, neuroimaging may be a valuable modality in CTE. Tau imaging using $\left[{ }^{8} \mathrm{~F}\right]$-Flortaucipir PET has shown promise to serve as a potential biomarker for CTE in vivo, although it may lack sensitivity in the early stages of the disease [11]. Aspecific features such as a degree of cortical atrophy (especially frontal lobes), ventricular enlargement, or increased prevalence of a cavum septum pellicidum may be revealed by anatomical imaging in the latter stages of the disease $[12,13]$.

Considerable debate exists as to what is driving the neurodegenerative disease and what is causing the latency in symptom onset [14]. In traumatic brain injury (TBI), neuroinflammation is thought to play an important role [15, 16]. Neuroinflammation refers to an inflammatory response in the brain which can involve the activation of microglia and astrocytes. This can be beneficial when, for example, pathogens are eliminated from the brain, such as in the case of stroke. However, in case of neurodegenerative diseases, microglia activation may become chronic and have a deleterious effect on the brain and thus on cognitive functioning of patients. This detrimental process, coined "immuno-excitotoxicty" [17], is thought to be triggered by repetitive head injury (RPI) resulting in axonal degeneration and microtubular degeneration $[17,18]$. In currently active athletes, cerebrospinal fluid (CSF) analysis of the triggering receptor on myeloid cells 2 (sTREM2) has been used as a means to detect activated microglia in vivo [19]. Disadvantages of using CSF as a biomarker of activated microglia are that it lacks information on the spatial distribution of activated microglia and that it requires a lumbar punction. From this perspective, neuroimaging may be a suitable less invasive alternative.
$18-\mathrm{kDa}$ translocator protein (TSPO) PET is the most widely studied research modality to image neuroinflammation in vivo. Increased expression of TSPO is mediated by activated microglia and reactive astrocites [20]. In TBI, TSPO PET has shown evidence of widespread microglial activation, which can persist even after 6 months [21]. TSPO imaging may be a valuable tool to assess the severity and location of TBI [22]. It has also been used in other types of CNS insults such as stroke. In stroke, TSPO PET was used to investigate the inflammatory response to ischemia in the core infarction region, peri-infarct zone, and in the contralateral hemisphere [23]. Increased expression of TSPO in currently active or recently retired athletes using PET was first described by Coughlin et al. in 2017 in NFL players (14 subjects and 16 controls) [24]. Since then, no additional reports have appeared assessing TSPO expression using PET in currently active athletes, particularly not in other contact sports. In this proof of principle study, we aim to assess the presence of neuroinflammation using $\left[{ }^{11} \mathrm{C}\right]-\mathrm{PK} 11195$ PET, a first-generation TSPO PET tracer, in competitive kickboxers. Kickboxing is a combat sport involving the use of punches and kicks with fists, knees, and elbows. Head injuries represent nearly half of injuries incurred by professional Muay-Thai boxers [25]. Competitive kickboxers are thus exposed to cumulative repeated injury to the head. Eleven rank A kickboxers and eleven controls underwent $\left[{ }^{11} \mathrm{C}\right] \mathrm{PK} 11195$ PET/CT scanning and MR imaging, whereas neuropsychological testing was performed to correlate and interpret imaging findings.

\section{Methods}

\section{Participants}

Competitive kickboxers with rank A and a minimum of 20 competitive bouts and 3 training sessions per week were recruited from gyms in the Netherlands. Rank A is the highest rank in kickboxing allowing participation in full contact bouts and can only be achieved after several years of intensive training. Kickboxers were not allowed to have fought a recent match or sustained a recent concussion (within 3 months of the scan). Healthy, age-matched controls without a history of TBI or participation in contact sports were recruited with local advertisements. All participants denied the use of anti-inflammatory drugs or anabolic steroids.

All subjects were asked to fill out two questionnaires: a quality of life scale (SF-36) [26], which is routinely used in the follow-up of mild TBI by the Department of Neurology of the University Medical Center Groningen, and the hospital anxiety and depression scale [27]. In addition, information was gathered in relation to sociodemography, medication, use of alcohol and cigarettes, disease history, history 
of mild TBI, and fighting record (wins-losses-draws). All subjects underwent neuropsychological testing, with tests for the following domains: intelligence (Dutch version of the national adult reading test [28]), mental speed and attention (trail making test (TMT) A and TMT B [29], Vienna test system (VTS) [30], the 15 words test [31] (both immediate and delayed recall (IR, DR)), digit span test [32], executive functioning (zoo map, subtest of the behavioral assessment of the dysexecutive syndrome (BADS [33]), Hayling test [34], controlled oral word association test (COWAT [35]), and emotion recognition (FEEST [36]). Laboratory testing included serum C-reactive protein (CRP) and interleukine-6 (IL-6) as peripheral markers of inflammation.

\section{Radiochemistry}

$\left[{ }^{11} \mathrm{C}\right]-\mathrm{PK} 11195$ was labeled by trapping ${ }^{11} \mathrm{C}$-methyl iodide in a solution of $1 \mathrm{mg}$ of $(R)$ - $N$-desmethyl-PK11195 and $10 \mathrm{mg}$ of potassium hydroxide in $300 \mu \mathrm{L}$ of dimethylsulfoxide. The reaction mixture was allowed to react for $1 \mathrm{~min}$ at $40{ }^{\circ} \mathrm{C}$, neutralized with $1 \mathrm{~mol} / \mathrm{L} \mathrm{HCl}$, and passed through a $45-\mu \mathrm{m}$ Millex-HV filter (Millipore). The filtrate was purified by high-performance liquid chromatography (HPLC) using a $\mu$ Bondapak C18 column $(7.8 \times 300 \mathrm{~mm})$ (Waters) with acetonitrile/25 mM NaH${ }_{2} \mathrm{PO}_{4}(\mathrm{pH} 3.5 ; 55 / 45)$ as the eluent (flow, $5 \mathrm{~mL} / \mathrm{min}$ ). To remove the organic solvents from the product, the collected HPLC fraction (retention time, $7 \mathrm{~min}$ ) was diluted with $100 \mathrm{~mL}$ of water and passed through an Oasis HLB 30-mg $(1 \mathrm{~mL})$ cartridge. The cartridge was washed twice with $10 \mathrm{~mL}$ of water and subsequently eluted with $1 \mathrm{~mL}$ of ethanol and $8 \mathrm{~mL}$ of water. The product was sterilized by filtration over a $0.20-\mu \mathrm{m}$ Millex-LG filter (Millipore). Quality control was performed by HPLC, using a Novapak C18 column $(150 \times 3.9 \mathrm{~mm})$ (Waters) with acetonitrile/25 $\mathrm{mM} \mathrm{NaH}_{2} \mathrm{PO}_{4}(\mathrm{pH} 3.5)(60 / 40)$ as the eluent at a flow of $1 \mathrm{~mL} / \mathrm{min}$. The radiochemical purity was always greater than $95 \%$. No differences were found between the net injected dose in healthy volunteers $(333 \pm 53 \mathrm{MBq})$ and that in patients $(336 \pm 27 \mathrm{MBq})(p=0.679)$.

\section{Imaging}

Subjects fasted for $6 \mathrm{~h}$ prior to the PET scan. $\left[{ }^{11} \mathrm{C}\right]-\mathrm{PK} 11195$ was injected with a speed of $0.5 \mathrm{~mL} / \mathrm{s}$ followed by a $60-\mathrm{min}$ dynamic PET scan consisting of 23 frames with increasing length on a Siemens Biograph mCT system (Siemens Medical Solutions USA, Inc.) with the head immobilized in a headrest to reduce motion artifacts. Images were reconstructed using Truex + TOF with 3 iterations and 21 subsets in a $400 \times 400$ matrix size (zoom 1.0). After intravenous injection of $\left[{ }^{11} \mathrm{C}\right]-\mathrm{PK} 11195$, arterial blood radioactivity was continuously monitored with an automated sampling system. In addition, 5 extra blood samples were collected at $10,20,30,45$, and $60 \mathrm{~min}$ after $\left[{ }^{11} \mathrm{C}\right]-\mathrm{PK} 11195$ injection to determine the amount of radioactivity in blood and plasma for calibration of the blood monitor, and to determine the amount of radioactive metabolites in plasma. MRI-T1w images were acquired for PET co-registration purposes. Subjects were scanned at a Philips Intera 3.0 T MRI scanner (Philips, Best, the Netherlands) with a 32-channel head coil. A 3D T1w TFE image was acquired for each subject using the following parameters: 160 sagittal slices without gap, FOV $(\mathrm{ap} \times \mathrm{rl} \times \mathrm{fh}) 256 \times 160 \times 256 \mathrm{~mm}$, acquired matrix $256 \times 256$, voxel size $1 \times 1 \times 1 \mathrm{~mm}$, repetition time $9 \mathrm{~ms}$, echo time $3.5 \mathrm{~ms}$, and flip angle 8 degrees. Diffusionweighted imaging (DWI) was performed using a single-shot spin-echo echo-planar imaging sequence with the following parameters: 65 axial slices of $2 \mathrm{~mm}$ without gap, FOV $240 \times 240 \times 130 \mathrm{~mm}^{3}$, acquisition matrix $117 \times 120$, reconstructed voxel size $1.88 \times 1.88 \times 2 \mathrm{~mm}^{3}$, TR $8877 \mathrm{~ms}$, TE $60 \mathrm{~ms}$, flip angle $90^{\circ}, 60$ diffusion directions (and seven non-diffusion-weighted scans averaged to one volume), and $b$-value 0 and $1000 \mathrm{~s} / \mathrm{mm}^{2}$. The noise of the diffusion scans was first reduced $[37,38]$ and corrected for Gibbs ringing artefacts [39]. This was followed by motion correction with correction for eddy-current induced distortions [40, 41] and bias field correction [42]. Subsequently, the diffusion data were subjected to diffusion tensor analysis using FMRIB Software Library (FSL v6.0.4.) [43].

All PET images were coregistered to the individual anatomical T1w MRI and spatially normalized to Montreal Neurological Institute (MNI) space using PMOD (version 4.0, PMOD Technologies Ltd, Zürich, Switzerland). Brain regions were defined using the Hammers maximum probability atlas (Hammers N3083) containing frontal lobes, parietal lobes, temporal lobes (with exception of the amygdala and the hippocampus, which were analyzed separately), occipital lobes, cerebellum, striatum, and white matter. In addition a whole brain grey matter (GM) region was generated, combining all cortical regions. For each patient, the binding potential $\left(B P_{\mathrm{ND}}\right)$ was calculated, defined as $k_{3} / k_{4}$, and used as outcome measure, using a 2 tissue compartment model with $K_{1} / K_{2}$ fixed to the value obtained for whole brain GM. For kinetic modeling, frame length-dependent weighting was used for fitting the time activity curve, a correction for blood delay was used, and blood data was fitted [44]. Fixing of $k_{4}$ to the whole brain GM value was performed in subregions when this parameter could not be adequately calculated (e.g., in case of a very high standard error (>25\%), occurring in 7 subjects). Group level comparisons were performed using independent sample Mann-Whitney $\mathrm{U}$ tests, with statistical threshold for significance set at 0.05 . A nonparametric test was chosen because it was anticipated that a non-normal distribution could be present in kickboxers with individual outliers in [11]C-PK11195 binding depending on the RPI exposure. For statistical analysis, SPSS was 
used (IBM Corp. Released 2015. IBM SPSS Statistics for Windows, Version 23.0. Armonk, NY: IBM Corp). Correlations between regional $B P_{\mathrm{ND}}$ and the scores on the neuropsychological testing and questionnaires were assessed using scatter plots and Spearman's rank correlation coefficients using GraphPad Prism version 7.02 for Windows (GraphPad Software, La Jolla California USA, www.graphpad.com).

Diffusion tensor imaging data were generated with FSL. The fractional anisotropy (FA) and mean diffusivity (MD) maps were spatially normalized to the ICBM-DTI-81 maximum probability maps [45] using PMOD. Atlas-based white matter regions were automatically defined. FA and MD values of predefined white matter regions (Fig. 1, corpus callosum (genu, body, splenium), anterior limb of internal capsule, posterior limb of internal capsule, retrolenticular part of the internal capsule, anterior corona radiata, superior corona radiata, posterior corona radiata, posterior thalamic radiation, superior longitudinal fasciculus) were measured.
Similar to previous DTI studies in sports related repetitive head injury, these white matter regions were selected in order to be sensitive to potential changes related to projections from widespread areas of the brain, especially long axonal projections [24]. Additional MRI sequences included SWI, T2w flair, and DTI. All MRI images were visually assessed by a neuroradiologist $(\mathrm{AvdH})$ for the presence of structural abnormalities.

\section{Results}

Thirteen kickboxers and 13 age-matched controls were included in the study. Due to technical problems related to the PET scanning procedure (PET reconstruction problem $(n=1)$, failure to place arterial lining $(n=3)), 11$ kickboxers and 11 controls remained for the final analysis. Subject characteristics are shown in Table 1 . The mean age was

Table 1 Subject characteristics

\begin{tabular}{|c|c|c|c|}
\hline & Boxers $(n=11)$ & Controls $(n=11)$ & $p$ value $(t$-test $)$ \\
\hline Mean age (years) & $31.1(6.5)$ & $26.6(6.1)$ & 0.10 \\
\hline Sex (male, \%) & $100 \%$ & $90 \%$ & 0.34 \\
\hline \multicolumn{4}{|l|}{ SF 36: } \\
\hline - Physical functioning & $99.5(1.6)$ & $99(2.1)$ & 0.56 \\
\hline - Role limitation (physical) & $90(31.6)$ & $92.5(23.7)$ & 0.84 \\
\hline - Bodily pain & $83(22.9)$ & $90.5(16.7)$ & 0.42 \\
\hline - General health & $83.9(11.9)$ & $83.5(14.9)$ & 0.95 \\
\hline - Vitality & $76.0(11.3)$ & $71.0(11.5)$ & 0.34 \\
\hline - Social functioning & $91.4(15.6)$ & $85.3(14.1)$ & 0.37 \\
\hline - Role limitation (mental) & $100(0)$ & $79.9(35.9)$ & 0.09 \\
\hline - Health changes & $55.0(10.5)$ & $57.5(12.1)$ & 0.63 \\
\hline - Mental health & $80.4(10.1)$ & $77.6(11.5)$ & 0.57 \\
\hline \multicolumn{4}{|l|}{ HADS: } \\
\hline - Anxiety & $3.8(2.4)$ & $5.0(2.7)$ & 0.28 \\
\hline - depression & $2.5(2.5)$ & $3.1(1.8)$ & 0.84 \\
\hline \multicolumn{4}{|l|}{ Peripheral inflammatory markers: } \\
\hline - IL-6 (pg/ml) & $0.9(0.8)$ & $1.4(0.8)$ & 0.12 \\
\hline - CRP (mg/L) & $0.6(0.3)$ & $2.1(4.9)$ & 0.32 \\
\hline Education* & $5.3(0.9)$ & $5.7(0.8)$ & 0.29 \\
\hline Smoking $(n)$ & 2 & 2 & 1.00 \\
\hline Bouts fought & $38.4(24.4)$ & - & - \\
\hline - Won & $27.3(20.3)$ & & \\
\hline - Lost & $9.5(5.4)$ & & \\
\hline - Drawn & $4.9(1.2)$ & & \\
\hline Years in training & $15.8(7.1)$ & - & - \\
\hline Frequency of training (per week) & $4.9(1.2)$ & - & - \\
\hline Time since last bout (months) & $15.8(15.2)$ & - & - \\
\hline Comorbidities & PTSS $(n=1)$ & - & \\
\hline
\end{tabular}

Data are displayed in means with the standard deviation. *(1 less than 6 grades' primary education, 26 grades' primary education, $\mathbf{3} 8$ grades' primary education, $\mathbf{4}$ vocational secondary education, $\mathbf{5}$ lower general secondary school, $\mathbf{6}$ higher general secondary school, pre-university school, 7 university) 
Fig. 1 Fused FA/3D T1w MRI image after normalizing to atlas space. The VOIs delineate the white matter regions included in the DTI analyses

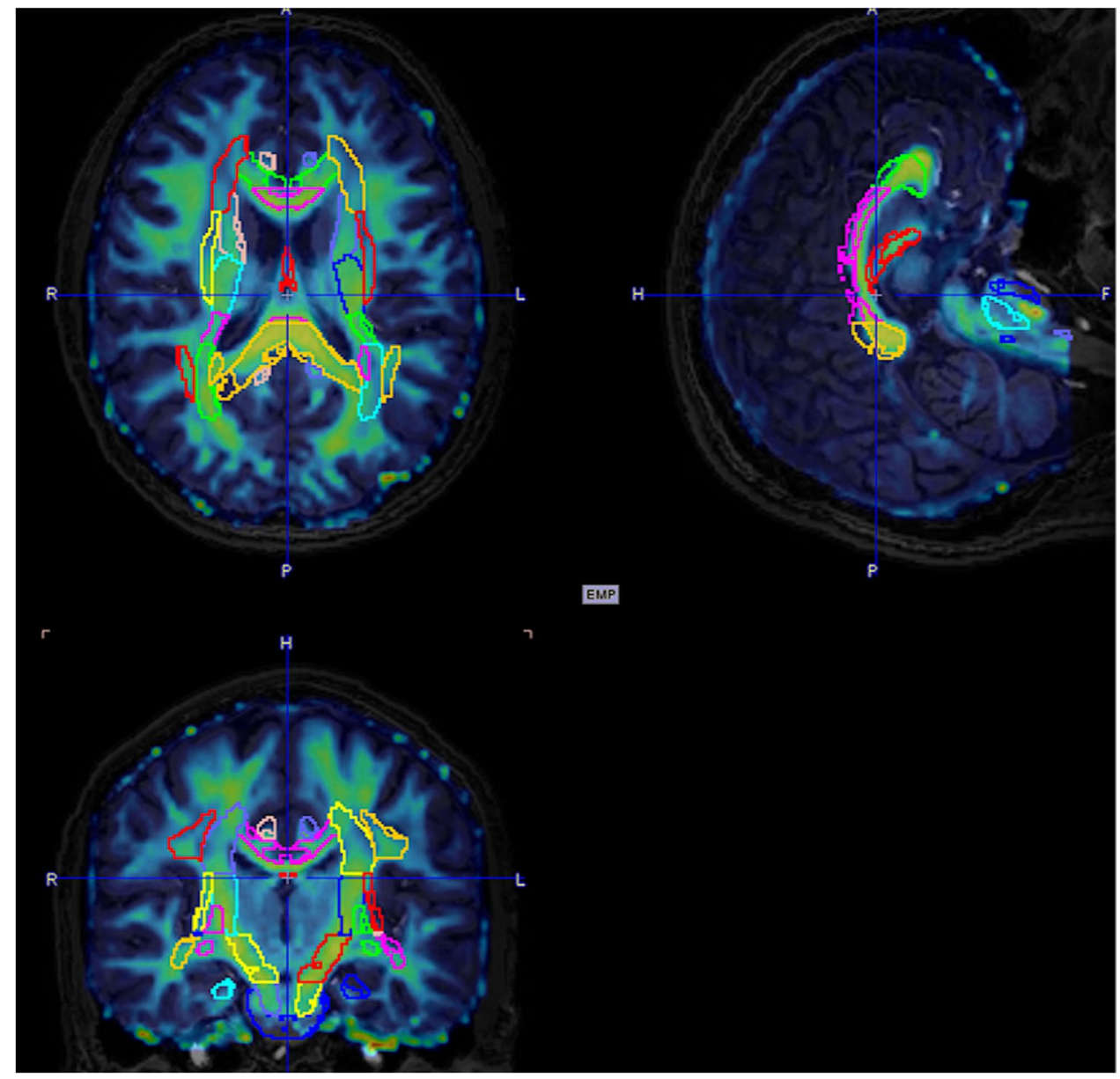

$31.1 \pm 1.9$ for the kickboxers and $26.6 \pm 1.8$ years for the control group $(p=0.10)$. No significant differences were observed in the SF-36 scores, education level, or inflammatory markers in serum. One kickboxer reported a posttraumatic stress disorder. Otherwise, no (psychiatric) comorbidities were present in both kickboxers and controls. One kickboxer had a history of participating in mixed martial arts. Regarding neuropsychological testing (Table 2), kickboxers displayed significantly faster reaction times in the VTS (RT-S1-DT, RT-S2-DT, RT-S3-DR). These are computerized determination tests which study attentional capacity and reaction speed among continuously changing acoustic and visual stimuli. Kickboxers performed worse on the TMT-B. The TMT is a test of executive functioning, and under the B condition, numbered as well as lettered circles have to be connected. Lower scores reflect lower cognitive flexibility [46]. No significant differences between subjects and controls in other tests were detected, although kickboxers tended to perform worse on the 15 word memory test.

On a group level, no statistically significant differences were found between the $B P_{\mathrm{ND}} \mathrm{s}$ in kickboxers and healthy controls (Table 3). Other relevant parameters of the analysis $\left(K_{l}, K_{l} / K_{2}\right)$ are shown in the supplemental data (Table S4,
Fig. S5). Individually, three kickboxers consequently showed high $B P_{\mathrm{ND}} \mathrm{S}$ in nearly all regions evaluated, with the highest $B P_{\mathrm{ND}}$ s being observed in the brainstem and the thalamus (Fig. 2). Without these outliers, medians were similar in both groups ( $B P$ nd whole brain 0.71 in kickboxers vs. 0.80 in controls, $p=0.90)$. Mean whole brain $k 3$ and $k 4$ were 0.21 and 0.06 in the kickboxers with high $B P$ nd vs. 0.02 and 0.03 in those with normal $B P$ nd ( $p=0.21$ and $0.44, T$-test). There was no correlation between the measured $B P_{\mathrm{ND}} \mathrm{s}$ of $\left[{ }^{11} \mathrm{C}\right]-\mathrm{PK} 11195$ in the whole brain and age, bouts fought, bouts lost, years training, and training frequency in kickboxers (Fig. 3). Significantly increased FA values were observed in the superior corona radiata (SCR) bilaterally in kickboxers compared with control subjects (SCR right 0.42 vs. 0.39 , $p=0.002$, left 0.44 vs. $0.41, p=0.006$ ). The measured FA and MD values in other white matters regions did not differ significantly between groups (Fig. 4, Table S5, supplemental data). One control showed a susceptibility artifact, interpreted as a microbleed, whereas none was present in the group of kickboxers. None of the participants showed T2 flair white matter abnormalities. In one kickboxer, a cavernous malformation was detected as an incidental finding. The three kickboxers with high WB $B P_{\text {nd }}$ s did not achieve 
Table 2 Results of neuropsychological tests

\begin{tabular}{lllll}
\hline & Boxers $(n=11)$ & Controls $(n=11)$ & $F$ & $\begin{array}{l}p \text { value } \\
\text { (ANOVA) }\end{array}$ \\
\hline Estimated premorbid IQ & $99.0(2.58)$ & $102.4(1.77)$ & 1.13 & 0.30 \\
15 word test: & & & \\
- Instant recall & $47.2(2.42)$ & $53.7(2.17)$ & 4.06 & 0.06 \\
- Delayed recall & $10.64(0.62)$ & $12.09(0.39)$ & 3.91 & 0.06 \\
Digit span: & & & & \\
- CR forward & $9.82(0.57)$ & $9.18(0.64)$ & 0.55 & 0.47 \\
- CR backward & $7.55(0.37)$ & $7.27(0.80)$ & 0.10 & 0.76 \\
VTS: & & & & \\
-RT-S1-DT & $238.1(10.12)$ & $278.1(10.52)$ & 7.51 & 0.01 \\
-RT-S2-DT & $197.0(6.63)$ & $233.3(9.34)$ & 10.03 & 0.005 \\
-RT-S3-DR & $325.1(12.81)$ & $394.8(13.52)$ & 14.02 & 0.001 \\
-DT-S1-correct & $235.1(6.31)$ & $241.9(6.35)$ & 0.58 & 0.46 \\
TMT-A & $27.60(4.32)$ & $26.18(3.00)$ & 0.08 & 0.79 \\
TMT-B & $71.0(8.65)$ & $50.36(3.91)$ & 4.72 & 0.04 \\
Zoo map (total score) & $11.09(1.86)$ & $13.27(0.82)$ & 1.15 & 0.30 \\
Hayling (overall scaled) & $6.40(0.34)$ & $5.91(0.37)$ & 0.95 & 0.34 \\
COWAT & $43.18(4.42)$ & $44.73(3.54)$ & 0.07 & 0.79 \\
FEEST & $49.09(1.56)$ & $49.45(1.34)$ & 0.03 & 0.86 \\
\hline
\end{tabular}

Data are displayed in means with the standard error of the mean

\begin{tabular}{lllll}
\hline Brain region & Kickboxers $(n=11)$ & Controls $(n=11)$ & $\begin{array}{l}p \text { value }(\text { Mann- } \\
\text { Whitney U) }\end{array}$ & Effect size r \\
\hline Brainstem & $1.60(0.80-3.54)$ & $0.98(0.76-1.34)$ & 0.22 & 0.39 \\
Frontal lobes & $1.08(0.56-2.39)$ & $0.68(0.60-0.93)$ & 0.40 & 0.26 \\
Hippocampus & $1.17(0.47-2.79)$ & $0.58(0.50-1.03)$ & 0.17 & 0.43 \\
Amygdala & $1.07(0.54-2.96)$ & $0.61(0.45-0.93)$ & 0.13 & 0.46 \\
Temporal lobes & $1.10(0.64-2.23)$ & $0.78(0.42-0.99)$ & 0.24 & 0.37 \\
Parietal lobes & $1.01(0.66-3.01)$ & $0.78(0.65-1.13)$ & 0.44 & 0.24 \\
Occipital lobes & $1.07(0.64-2.76)$ & $0.85(0.68-1.10)$ & 0.37 & 0.28 \\
Insula and cingulate gyri & $1.03(0.66-3.01)$ & $0.66(0.48-0.83)$ & 0.06 & 0.58 \\
Thalamus & $1.21(0.77-2.50)$ & $0.90(0.79-1.32)$ & 0.37 & 0.29 \\
Striatum & $1.06(0.57-2.16)$ & $0.67(0.55-0.86)$ & 0.22 & 0.37 \\
Cerebellum & $0.99(0.59-3.10)$ & $0.84(0.51-1.00)$ & 0.30 & 0.33 \\
White matter & $1.29(0.74-2.60)$ & $0.94(0.88-1.41)$ & 0.52 & 0.21 \\
Whole brain & $1.15(0.62-2.60)$ & $0.80(0.53-1.03)$ & 0.24 & 0.36 \\
\hline
\end{tabular}

Table 3 Median binding potential $\left(B P_{\mathrm{ND}}\right.$ and interquartile ranges) of $\left[{ }^{11} \mathrm{C}\right]-\mathrm{PK} 11195$ PET in kickboxers and controls scores below the 5 th or higher than the 95 th percentile on neuropsychological testing. No significant correlations were observed between the results on neuropsychological testing and regional $B P_{\mathrm{ND}} \mathrm{s}$ in kickboxers.

\section{Discussion}

In this study, no significantly increased whole brain $B P_{\mathrm{ND}} \mathrm{S}$ of $\left[{ }^{11} \mathrm{C}\right]-\mathrm{PK} 11195$ were observed in a group of highly competitive kickboxers in comparison with healthy controls.
Individually, three kickboxers showed high $B P_{\text {nd }}$ values in widespread brain regions, outside the range typically reported in healthy subjects, both in the current study and in previous studies [44]. Despite negative results on a group level, these findings seem to confirm that a degree of neuroinflammation may be present in currently active athletes without relationship to a recent (within three months) mild TBI. Thus, the widespread increased TSPO PET signal in these individuals could reflect the early consequences of RHI incurred during their career. The current study adds important information to the very limited amount of in vivo 
Fig. 2 BPnd' of $\left[{ }^{11} \mathrm{C}\right]-\mathrm{PK} 11195$ in kickboxers $(n=11)$ and controls $(n=11)$. The symbols represent individual subjects. The horizontal bar reflects the median and the error bars the 1st and 3rd quartile
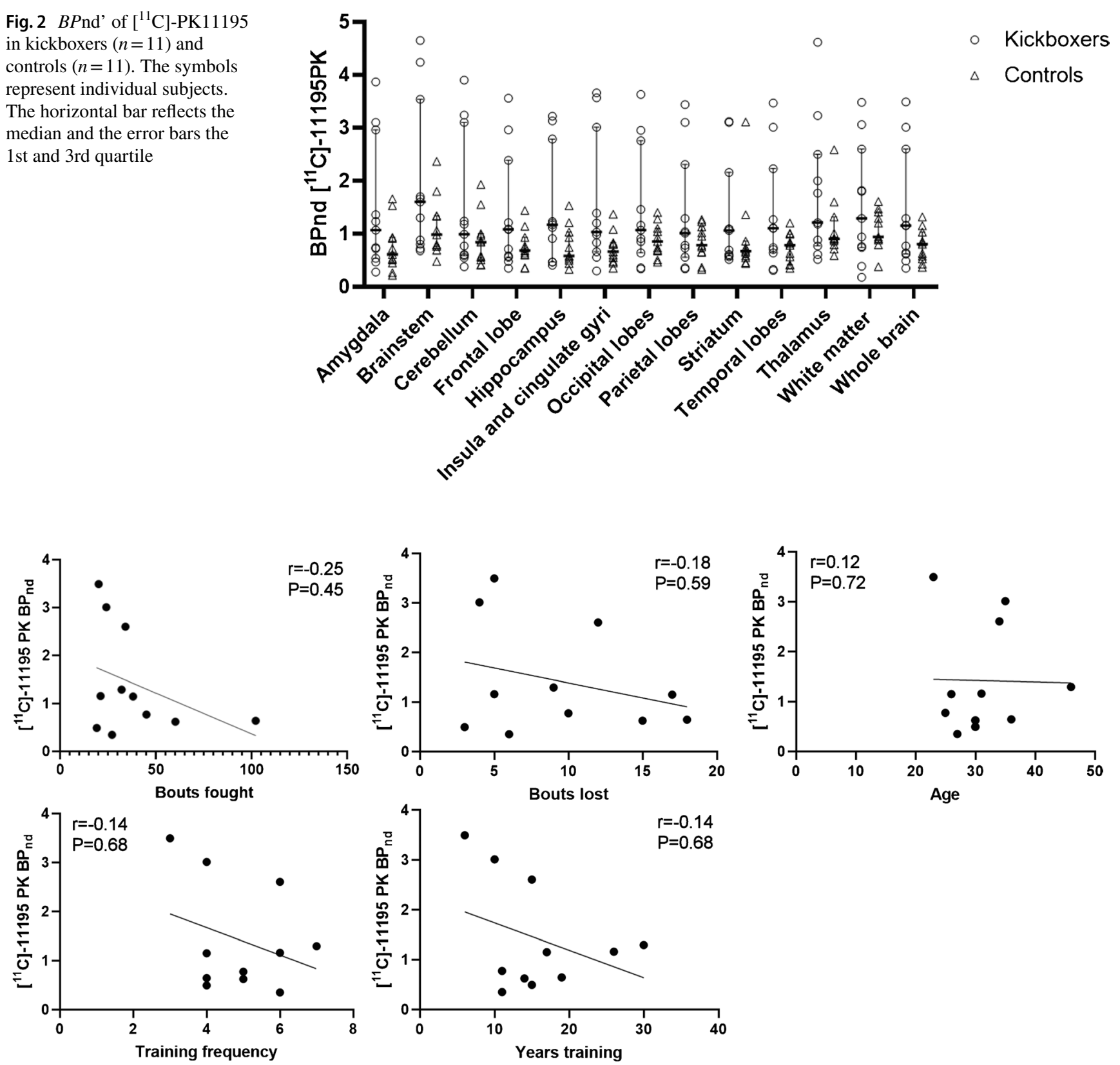

Fig. 3 Scatter plots showing the relation between the whole brain BPnds of $\left[{ }^{11} \mathrm{C}\right]-\mathrm{PK} 11195$ and bouts fought, bouts lost, age, training frequency, and years training. Spearman's rank correlation coefficient $r$ is displayed in each graph

neuroimaging studies available targeted at microglial activation in contact sports athletes and is the first of its kind conducted in subjects participating in other sports than American football.

Previously, Coughlin and colleagues reported increased TSPO PET signal in multiple brain regions in currently active and recently retired NFL players, especially in the supramarginal gyrus [24], which was positively correlated with years of play. A similar relationship has been established in pathology studies, with a study in American football players $(n=66)$ reporting an association between the level of CD68 positivity and the number of years of playing football [47]. Years of play has also been linked with the risk of developing CTE, with an estimated doubling of risk after every 2.6 years of play, and increased NFT burden [48]. In our study, however, the upregulation of TSPO — as measured by the increased $B P_{n d} \mathrm{~s}$ - could not be correlated to the number of years training, training frequency, number of bouts fought and lost, or fighting style. On a group level, kickboxers showed superior performance on reaction tests evaluating basal mental speed. Previously, various psychological skills have been associated with increased performance in 
kickboxing, including relaxation and controlling anxiety, as well as personal characteristics such as mental toughness [49]. The 3 kickboxers with high $B P_{\mathrm{nd}} \mathrm{s}$ in widespread regions of the brain obtained scores within the 5th and 95th percentile on each test. Coughlin and colleagues similarly did not report significant differences in the performance on neuropsychiatric tests between athletes and controls despite evidence of TSPO upregulation [24]. This could be related to the relatively young age of the subjects included, as some studies indicated that aging is associated with increased prevalence of $\mathrm{MCI}$ and neurodegenerative disease in former contact athletes [50].

Evidence of activated microglia in athletes exposed to RHI has been less consistently reported in studies using CSF measurements. A previous study evaluating the levels of sTREM2 (a marker of microglia activation) in the CSF failed to find significant differences between symptomatic former NFL players $(n=68)$ and controls [19]. In the same study, a positive correlation between sTREM 2 and p-tau was seen, which led to authors to suggest that re-activation of microglia may occur in the setting of neurodegeneration. The activation of microglia as measured with PET in a subset of kickboxers in our study may reflect a preclinical state associated with the development of cognitive, affective, and behavioral changes later in life, but this potential relationship remains to be fully elucidated. Future studies using TSPO PET may benefit from a longitudinal design to investigate the course of TSPO upregulation in athletes and a potential relationship with the onset of neuropsychiatric symptoms.

In our study, three boxers showed high $B P_{\text {nd }}$ values in nearly all regions evaluated. The pattern of diffuse neuroinflammation is in accordance with the neuronal deficits detected using functional imaging techniques such as $\left[{ }^{18} \mathrm{~F}\right]$ FDG PET, as a measure of brain metabolism, and perfusion SPECT in widespread areas of the brain. Provenzano et al. observed decreased metabolism in the posterior cingulate cortex, parieto-occipital, frontal lobes, and cerebellum in boxers (mean age 30) [51]. Amen and colleagues reported perfusion deficits in prefrontal, temporal, parietal, and occipital lobes and cerebellar regions in American football players
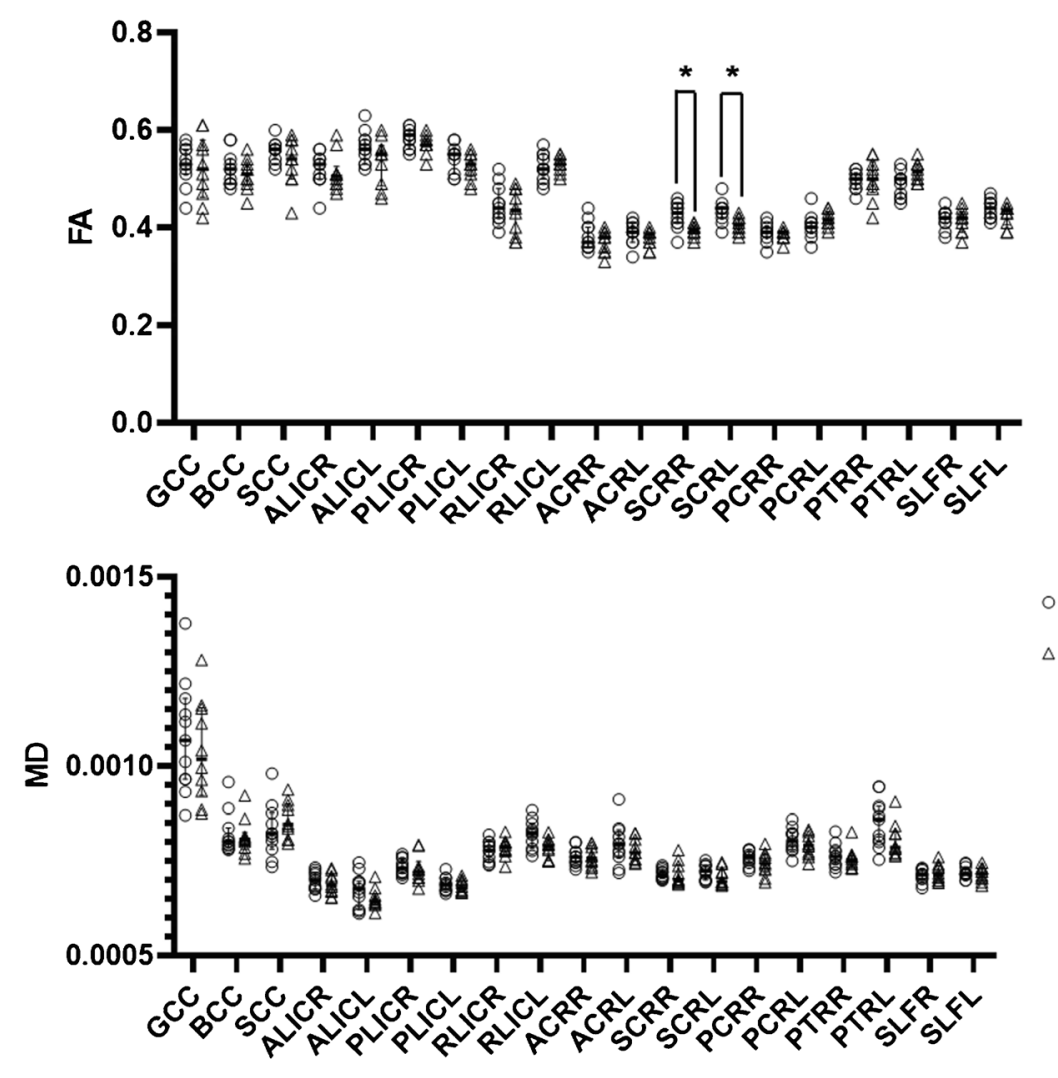

- Kickboxers
$\triangle$ Controls

- Kickboxers

$\triangle$ Controls
Fig. 4 FA and MD values of kickboxers and controls in white matter regions. The symbols represent individual subjects. The horizontal bar reflects the median and the error bars the 1 st and 3rd quartile. GCC genu corpus callosum, BCC body corpus callosum, SCC splenium corpus callosum, ALIC R anterior limb of internal capsule right, ALIC L anterior limb of internal capsule left, PLIC R posterior limb of internal capsule right, PLIC $\mathbf{L}$ posterior limb of internal capsule left, RIC R retrolenticular part of internal capsule right, RIC
$\mathbf{L}$ retrolenticular part of internal capsule left, ACR $\mathbf{R}$ anterior corona radiata right, ACR $\mathbf{L}$ anterior corona radiata left, SCR R superior corona radiata right, SCR $\mathbf{L}$ superior corona radiata left, PCR R posterior corona radiata right, PCR $\mathbf{L}$ posterior corona radiata left, PTL $\mathbf{R}$ posterior thalamic radiation right, $\mathbf{P T R} \mathbf{L}$ posterior thalamic radiation left, SLF $\mathbf{R}$ superior longitudinal fasciculus right, SLF $\mathbf{L}$ superior longitudinal fasciculus left. *Significant difference 
(mean age 52) [52]. Interestingly in our study, kickboxers showed increased whole brain $K_{1}$ values, which is indicative of relatively enhanced perfusion. Although speculative, this may be due to the lack of control for physical exercise in the control group. Previously, exercise has been associated with increased resting state cerebral perfusion in multiple areas of the brain in athletes [53]. Related to TBI, widespread areas of increased $\left[{ }^{11} \mathrm{C}\right]-\mathrm{PK} 11195$ have also been reported, including areas unaffected on structural MRI [21].

Significantly increased FA values in the superor corona radiata were observed in kickboxers in comparison to controls. DTI measurements in other regions did not differ significantly between groups. Typical features of the microstructural damage previously described in professional boxers included reduced FA and increased MD in vulnerable brain regions in the white matter [54, 55], although negative results have also been reported [56]. Abnormally high FA values in the superior corona radiata were previously described in the context of mild traumatic brain injury by Mayer et al., who attributed this finding to cytotoxic edema or changes in water content within the myelin sheath [57]. A methodological drawback of some of the previous DTI reports is the manual drawing of VOIs, which we tried to overcome using an automatic atlas based approach. The lack of typical positive findings in our study may be a consequence of the inclusion of asymptomatic kickboxers and relatively young age.

\section{Limitations}

In this study, years training, bouts fought, bouts lost, and training frequency were used as a surrogate marker of exposure to repetitive head injury. This is however not a perfect estimate, and individual variation, e.g., training intensity, may have resulted in undetected differences related to exposure. In addition, all subjects included were without any clinical complaints and relatively young, which may have reduced the a priori chances of increased TSPO binding. Second, $\left[{ }^{11} \mathrm{C}\right]-\mathrm{PK} 11195$ is a first-generation TSPO PET tracer, which may lack sensitivity in comparison to newer ligands. On the other hand, $\left[{ }^{11} \mathrm{C}\right]-\mathrm{PK} 11195$ has not been linked to genetically prone differences in binding affinity, an issue which requires genotyping when using second generation TSPO tracers [58]. Finally, the study was cross-sectional; therefore, longitudinal information related to TSPO binding and the potential onset of complaints is lacking.

\section{Conclusion}

The current study fails to provide evidence of TSPO upregulation in currently active kickboxers on a group level, but the widespread increased binding of $\left[{ }^{11} \mathrm{C}\right]-\mathrm{PK} 11195$ in approximately 1 out of 4 kickboxers suggests that neuroinflammation may be present as a consequence of repetitive head injury. In this group of asymptomatic kickboxers, this upregulation could not be correlated with the results on neuropsychological testing or DTI MRI findings. Future studies using a longitudinal design may determine whether the observed TSPO upregulation in active athletes is related to the development of neuropsychiatric symptoms.

Supplementary Information The online version contains supplementary material available at https://doi.org/10.1007/s00259-022-05715-x.

Acknowledgements We would like to thank all participants who volunteered to take part of this study, particularly the rank A kickboxers from various gyms in the Netherlands.

\section{Declarations}

Ethics approval Written informed consent was given by each individual, and the study was approved by the local ethics committee.

Consent for publication Not applicable.

Conflict of interest The authors declare no competing interests.

Open Access This article is licensed under a Creative Commons Attribution 4.0 International License, which permits use, sharing, adaptation, distribution and reproduction in any medium or format, as long as you give appropriate credit to the original author(s) and the source, provide a link to the Creative Commons licence, and indicate if changes were made. The images or other third party material in this article are included in the article's Creative Commons licence, unless indicated otherwise in a credit line to the material. If material is not included in the article's Creative Commons licence and your intended use is not permitted by statutory regulation or exceeds the permitted use, you will need to obtain permission directly from the copyright holder. To view a copy of this licence, visit http://creativecommons.org/licenses/by/4.0/.

\section{References}

1. McKee AC, Cantu RC, Nowinski CJ, Hedley-Whyte ET, Gavett $\mathrm{BE}$, Budson AE, et al. Chronic traumatic encephalopathy in athletes: progressive tauopathy after repetitive head injury. J Neuropathol Exp Neurol. 2009;68(7):709-35.

2. McKee AC, Stern RA, Nowinski CJ, Stein TD, Alvarez VE, Daneshvar DH, et al. The spectrum of disease in chronic traumatic encephalopathy. Brain. 2013;136(Pt 1):43-64.

3. Turk KW, Budson AE. Chronic traumatic encephalopathy. Continuum (Minneap Minn). 2019;25(1):187-207.

4. Omalu BI, Hamilton RL, Kamboh MI, DeKosky ST, Bailes J. Chronic traumatic encephalopathy (CTE) in a national football league player: case report and emerging medicolegal practice questions. J Forensic Nurs 2010 Spring;6(1):40-46.

5. Omalu BI, Bailes J, Hammers JL, Fitzsimmons RP. Chronic traumatic encephalopathy, suicides and parasuicides in professional American athletes: the role of the forensic pathologist. Am J Forensic Med Pathol. 2010;31(2):130-2.

6. McKee AC, Robinson ME. Military-related traumatic brain injury and neurodegeneration. Alzheimers Dement. 2014;10(3 Suppl):S242-53. 
7. Gavett BE, Stern RA, McKee AC. Chronic traumatic encephalopathy: a potential late effect of sport-related concussive and subconcussive head trauma. Clin Sports Med 2011;30(1):179-88, xi.

8. McKee AC, Cairns NJ, Dickson DW, Folkerth RD, Keene CD, Litvan I, et al. The first NINDS/NIBIB consensus meeting to define neuropathological criteria for the diagnosis of chronic traumatic encephalopathy. Acta Neuropathol. 2016;131(1):75-86.

9. Mez J, Daneshvar DH, Kiernan PT, Abdolmohammadi B, Alvarez VE, Huber BR, et al. Clinicopathological evaluation of chronic traumatic encephalopathy in players of American football. JAMA. 2017;318(4):360-70.

10. Katz DI, Bernick C, Dodick DW, Mez J, Mariani ML, Adler CH, et al. National Institute of Neurological Disorders and Stroke Consensus Diagnostic criteria for traumatic encephalopathy syndrome. Neurology. 2021;96(18):848-63.

11. Lesman-Segev OH, La Joie R, Stephens ML, Sonni I, Tsai R, Bourakova V, et al. Tau PET and multimodal brain imaging in patients at risk for chronic traumatic encephalopathy. Neuroimage Clin. 2019;24:102025.

12. Little DM, Geary EK, Moynihan M, Alexander A, Pennington $\mathrm{M}$, Glang $\mathrm{P}$, et al. Imaging chronic traumatic brain injury as a risk factor for neurodegeneration. Alzheimers Dement. 2014;10(3 Suppl):S188-95.

13. Koerte IK, Hufschmidt J, Muehlmann M, Tripodis Y, Stamm JM, Pasternak O, et al. Cavum Septi Pellucidi in symptomatic former professional football players. J Neurotrauma. 2016;33(4):346-53.

14. Kriegel J, Papadopoulos Z, McKee AC. Chronic traumatic encephalopathy: is latency in symptom onset explained by tau propagation? Cold Spring Harb Perspect Med 2018;8(2):https:// doi.org/10.1101/cshperspect.a024059.

15. Werner C, Engelhard K. Pathophysiology of traumatic brain injury. Br J Anaesth. 2007;99(1):4-9.

16. Gaetz M. The neurophysiology of brain injury. Clin Neurophysiol. 2004;115(1):4-18.

17. Blaylock RL, Maroon J. Immunoexcitotoxicity as a central mechanism in chronic traumatic encephalopathy-a unifying hypothesis. Surg Neurol Int 2011;2:107-7806.83391.

18. Tharmaratnam T, Iskandar MA, Tabobondung TC, Tobbia I, Gopee-Ramanan P, Tabobondung TA. Chronic traumatic encephalopathy in professional American football players: where are we now? Front Neurol. 2018;9:445.

19. Alosco ML, Tripodis Y, Fritts NG, Heslegrave A, Baugh $\mathrm{CM}$, Conneely S, et al. Cerebrospinal fluid tau, Abeta, and sTREM2 in former national football league players: modeling the relationship between repetitive head impacts, microglial activation, and neurodegeneration. Alzheimers Dement. 2018;14(9):1159-70.

20. Rupprecht R, Papadopoulos V, Rammes G, Baghai TC, Fan J, Akula N, et al. Translocator protein (18 kDa) (TSPO) as a therapeutic target for neurological and psychiatric disorders. Nat Rev Drug Discov. 2010;9(12):971-88.

21. Folkersma H, Boellaard R, Yaqub M, Kloet RW, Windhorst AD, Lammertsma AA, et al. Widespread and prolonged increase in (R)-(11)C-PK11195 binding after traumatic brain injury. J Nucl Med. 2011;52(8):1235-9.

22. Dimitrova-Shumkovska J, Krstanoski L, Veenman L. Diagnostic and therapeutic potential of TSPO studies regarding neurodegenerative diseases, psychiatric disorders, alcohol use disorders, traumatic brain injury, and stroke: an update. Cells 2020;9(4):https:// doi.org/10.3390/cells9040870.

23. Price CJ, Wang D, Menon DK, Guadagno JV, Cleij M, Fryer $\mathrm{T}$, et al. Intrinsic activated microglia map to the periinfarct zone in the subacute phase of ischemic stroke. Stroke. 2006;37(7):1749-53.
24. Coughlin JM, Wang Y, Minn I, Bienko N, Ambinder EB, Xu X, et al. Imaging of glial cell activation and white matter integrity in brains of active and recently retired national football league players. JAMA Neurol. 2017;74(1):67-74.

25. Gartland S, Malik MH, Lovell ME. Injury and injury rates in Muay Thai kick boxing. Br J Sports Med. 2001;35(5):308-13.

26. Brazier JE, Harper R, Jones NM, O'Cathain A, Thomas KJ, Usherwood T, et al. Validating the SF-36 health survey questionnaire: new outcome measure for primary care. BMJ. 1992;305(6846):160-4.

27. Zigmond AS, Snaith RP. The hospital anxiety and depression scale. Acta Psychiatr Scand. 1983;67(6):361-70.

28. Schmand B, Bakker D, Saan R, Louman J. The Dutch reading test for adults: a measure of premorbid intelligence level. Tijdschr Gerontol Geriatr. 1991;22(1):15-9.

29 Reitan RM. The relation of the trail making test to organic brain damage. J Consult Psychol. 1955;19(5):393-4.

30. Schuhfried G. Vienna test system: psychological assessment. 2013.

31. Green P, Lees-Haley P, III L. The word memory test and the validity of neuropsychological test scores. Journal of Forensic Neuropsychology 2003 01;2.

32. Blackburn HL, Benton AL. Revised administration and scoring of the digit span test. J Consult Psychol. 1957;21(2):139-43.

33. Burgess PW, Alderman N, Evans J, Emslie H, Wilson BA. The ecological validity of tests of executive function. J Int Neuropsychol Soc. 1998;4(6):547-58.

34. Burgess PW, Shallice T. The Hayling and Brixton Tests. 1997.

35. Schmand B, Groenink SC, van den Dungen M. Letter fluency: psychometric properties and Dutch normative data. Tijdschr Gerontol Geriatr. 2008;39(2):64-76.

36. Facial expressions of emotion: Stimuli and tests (FEEST); 2002.

37. Cordero-Grande L, Christiaens D, Hutter J, Price AN, Hajnal JV. Complex diffusion-weighted image estimation via matrix recovery under general noise models. Neuroimage. 2019;200:391-404.

38. Veraart J, Novikov DS, Christiaens D, Ades-Aron B, Sijbers J, Fieremans E. Denoising of diffusion MRI using random matrix theory. Neuroimage. 2016;142:394-406.

39. Kellner E, Dhital B, Kiselev VG, Reisert M. Gibbs-ringing artifact removal based on local subvoxel-shifts. Magn Reson Med. 2016;76(5):1574-81.

40. Andersson JLR, Sotiropoulos SN. An integrated approach to correction for off-resonance effects and subject movement in diffusion MR imaging. Neuroimage. 2016;125:1063-78.

41. Andersson JLR, Graham MS, Zsoldos E, Sotiropoulos SN. Incorporating outlier detection and replacement into a non-parametric framework for movement and distortion correction of diffusion MR images. Neuroimage. 2016;141:556-72.

42. Tustison NJ, Avants BB, Cook PA, Zheng Y, Egan A, Yushkevich PA, et al. N4ITK: improved N3 bias correction. IEEE Trans Med Imaging. 2010;29(6):1310-20.

43. Smith SM, Jenkinson M, Woolrich MW, Beckmann CF, Behrens TE, Johansen-Berg H, et al. Advances in functional and structural MR image analysis and implementation as FSL. Neuroimage. 2004;23(Suppl 1):S208-19.

44. Kropholler MA, Boellaard R, Schuitemaker A, van Berckel BN, Luurtsema G, Windhorst AD, et al. Development of a tracer kinetic plasma input model for (R)-[11C]PK11195 brain studies. J Cereb Blood Flow Metab. 2005;25(7):842-51.

45. Mori S, Oishi K, Jiang H, Jiang L, Li X, Akhter K, et al. Stereotaxic white matter atlas based on diffusion tensor imaging in an ICBM template. Neuroimage. 2008;40(2):570-82.

46. Arbuthnott K, Frank J. Trail making test, part B as a measure of executive control: validation using a set-switching paradigm. $\mathrm{J}$ Clin Exp Neuropsychol. 2000;22(4):518-28. 
47. Cherry JD, Tripodis Y, Alvarez VE, Huber B, Kiernan PT, Daneshvar DH, et al. Microglial neuroinflammation contributes to tau accumulation in chronic traumatic encephalopathy. Acta Neuropathol Commun 2016;4(1):112-016-0382-8.

48. Mez J, Daneshvar DH, Abdolmohammadi B, Chua AS, Alosco ML, Kiernan PT, et al. Duration of American football play and chronic traumatic encephalopathy. Ann Neurol. 2020;87(1):116-31.

49. Slimani M, Chaabene H, Miarka B, Franchini E, Chamari K, Cheour F. Kickboxing review: anthropometric, psychophysiological and activity profiles and injury epidemiology. Biol Sport. 2017;34(2):185-96.

50. Schaffert J, LoBue C, Fields L, Wilmoth K, Didehbani N, Hart J $\mathrm{Jr}$, et al. Neuropsychological functioning in ageing retired NFL players: a critical review. Int Rev Psychiatry. 2020;32(1):71-88.

51. Provenzano FA, Jordan B, Tikofsky RS, Saxena C, Van Heertum RL, Ichise M. F-18 FDG PET imaging of chronic traumatic brain injury in boxers: a statistical parametric analysis. Nucl Med Commun. 2010;31(11):952-7.

52. Amen DG, Willeumier K, Omalu B, Newberg A, Raghavendra C, Raji CA. Perfusion Neuroimaging abnormalities alone distinguish national football league players from a healthy population. J Alzheimers Dis. 2016;53(1):237-41.
53. Alfini AJ, Weiss LR, Leitner BP, Smith TJ, Hagberg JM, Smith JC. Hippocampal and cerebral blood flow after exercise cessation in master athletes. Front Aging Neurosci. 2016;8:184.

54. Chappell MH, Ulug AM, Zhang L, Heitger MH, Jordan BD, Zimmerman RD, et al. Distribution of microstructural damage in the brains of professional boxers: a diffusion MRI study. J Magn Reson Imaging. 2006;24(3):537-42.

55. Zhang L, Heier LA, Zimmerman RD, Jordan B, Ulug AM. Diffusion anisotropy changes in the brains of professional boxers. AJNR Am J Neuroradiol. 2006;27(9):2000-4.

56. Wilde EA, Hunter JV, Li X, Amador C, Hanten G, Newsome MR, et al. Chronic effects of boxing: diffusion tensor imaging and cognitive findings. J Neurotrauma. 2016;33(7):672-80.

57. Mayer AR, Ling J, Mannell MV, Gasparovic C, Phillips JP, Doezema D, et al. A prospective diffusion tensor imaging study in mild traumatic brain injury. Neurology. 2010;74(8):643-50.

58. Crawshaw AA, Robertson NP. The role of TSPO PET in assessing neuroinflammation. J Neurol. 2017;264(8):1825-7.

Publisher's note Springer Nature remains neutral with regard to jurisdictional claims in published maps and institutional affiliations. 